\section{Video-assisted thoracic surgery techniques for lung cancer: which is better?}

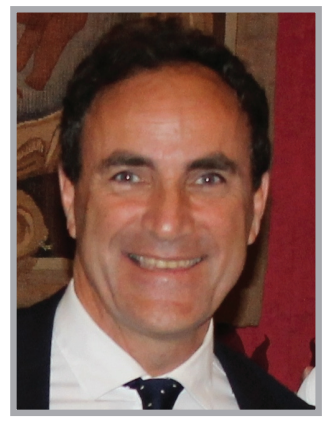

Marcello Migliore*

\author{
"The search for minimal invasiveness has a \\ prominent key role in thoracic surgery, but \\ research is vital to reveal the 'true' advantages of \\ surgical innovations.”
}

First draft submitted: 17 October 2016; Accepted for publication: 25 October 2016; Published online: 25 November 2016

\section{3rd Mediterranean Symposium on Thoracic Surgical Oncology, Catania, Italy, 21-22 April 2016}

The main aim of the 3rd Mediterranean Symposium in oncologic thoracic surgery was to discuss the role of video-assisted thoracic surgery (VATS) in the treatment of lung cancer, and to try to understand if the known widespread use in the common practice of thoracic surgery, including major resection for lung cancer, will influence the treatment of lung cancer. Secondary aims were an in-depth analysis of $\mathrm{N} 2$ disease in the era of minimally invasive surgery, and how to face the need of randomized trials in lung cancer.

In general the majority of the participating surgeons (Figure 1), agreed that the most important recent innovation in thoracic surgery is certainly the fast introduction of the so-called uniportal VATS (or single-incision VATS) to perform anatomical lung resection for lung cancer. Furthermore, it has been noted that most operations are performed in China, especially in Shanghai where surgeons are performing around 8000 lung resections per year.

\section{Problem statement}

Over the last 15 years, despite considerable innovation in VATS for anatomical lung resection has been introduced, several VATS modifications to perform anatomical resection for lung cancer have been reported as shown in Box 1. Interestingly, leaders of each technique try to convince other colleagues that their VATS technique is better than the other. Moreover, the rapid growth of VATS has resulted in some conceptual confusion about the type of VATS technique to use to perform anatomical lung resection, which has made it difficult for all but the most avid surgeons to keep up with developments in this domain.

Therefore, the purpose of this article is to critically examine all papers related to VATS published in this supplement in order to look at the extent to which recent innovation in VATS could influence the behavior of the surgeons; to provide pragmatic recommendations for
*Section of Thoracic Surgery, Department of General Surgery \& Medical Specialities, University of Catania, Policlinico University Hospital, Catania, Italy; mmiglior@unict.it

\section{KEYWORDS}

- lung cancer $\bullet$ lung resection

- video-assisted thoracic surgery 


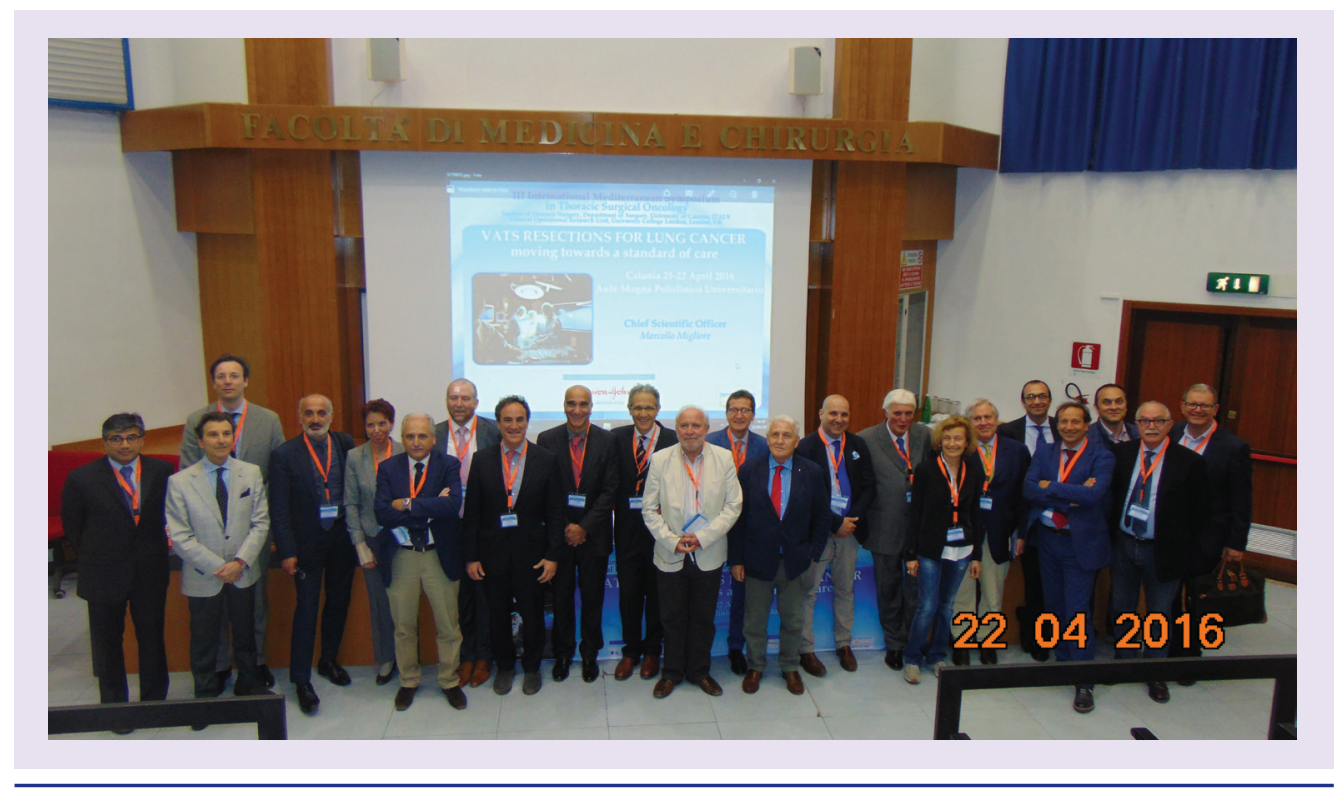

Figure 1. Faculty from the 3rd Mediterranean Symposium in Thoracic Surgical Oncology.

how to select the appropriate VATS technique for anatomical lung resection for lung cancer; and identify several interesting directions for future research.

\section{Semantic terminology}

The first attempt to clarify terminology was given 15 years ago [1]. Although recently Treasure wrote that VATS should stand for video-assisted thoracoscopic surgery and not for video-assisted thoracic surgery [2], I would disagree. The reason why I disagree is basically explained by the fact that video-thoracoscopic surgery should be used when a classic thoracoscope (optic and operative channel assembled in one instrument - medical thoracoscopy) is employed, while when the optic is separated from surgical instruments the term 'videoassisted thoracic surgery' better describes the method. In a few words, the $\mathrm{T}$ could create confusion as it could stay either for thoracoscopic or thoracic. It would be polite to call video-assisted thoracic surgery (VATS) and video-assisted thoracoscopic surgery (VAtS medical thoracoscopy).

Another point of discussion, but without an answer yet, is the weakness of the rationale of why a surgeon sometimes, during a VATS resection, looks directly inside the chest through a $5 \mathrm{~cm}$ utility incision with the help of an optic, the procedure should not be called VATS.

We have been very blessed that, with the intellectual inputs of international eminent thoracic surgeons, we have attempted to clarify and resolve the existing ambiguity in VATS procedures and propose specific terms identifying distinct approaches for minimally invasive thoracic procedures [3].

\section{Several VATS modifications for the same operation}

One thing is certain, whether the surgeon uses one VATS technique instead of another to treat lung cancer, he performs the same operation: lung resection and lymphadenectomy. The surgeon can prefer the fissureless method or another, but at the end of the story the same operation is performed. One could argue that single-incision VATS has less possibility to develop postoperative chest pain but this has not been demonstrated yet [4].

\section{N2 disease in the minimally invasive era}

Treasure and De Leyn [5] made an in-depth analysis of the new perception in the era of VATS to operate on patients with lung cancer if the N2 disease could be removed [6]. Nevertheless they pointed out that the European Society Thoracic Surgeons guidelines for intraoperative lymph node staging in non-small-cell lung cancer although called 'guidelines' fall short of the international standard required of clinically trustworthy 'guidelines'. The author proposes an analogy with breast cancer and suggests that randomized trials will be required rather than follow-up studies to obtain a definitive answer. 


\section{Robotic-assisted thoracic surgery}

During the symposium very little was discussed about robotic-assisted thoracic surgery (RATS) for lung cancer, and the main reason was due principally because in the real world, RATS is performed only in few worldwide centers, and controversy remains about the application of RATS because of the lack of well-established evidence. For the majority of hospitals, it is still too expensive, and the reported long duration of the operating room usage is not suistanable [7].

\section{Awake (tubeless) thoracic surgery}

Awake thoracic surgery has successfully been used to perform wedge resections of the lung for diagnostic purposes, pneumothorax and tracheal resections, but recently its use for major lung resection could arise ethical concern [8]; on the contrary, the concept of enhanced recovery ('fast-track') after thoracic surgery and anesthesia permits the allowance of an increased number of video-assisted parenchymal lung resections in managing primary lung cancer. A tremendous experience with 1000 tubeless VATS at the Rome Tor Vergata University demonstrated that this technique in expert hands can be accomplished in a safe manner with effective results [9].

VATS for lung cancer \& long-term survival The most wanted desire of a patient with cancer who undergoes an operation is to be cured, or, if it is not possible to be cured, to live longer with a good quality of life. The first question which arises is obvious: would VATS permit to survive longer than open surgery? During the symposium it was well shown, through a comparative analysis using SEER-Medicare database $[10,11]$ that VATS is 'probably' better than open surgery but the question of "Is VATS superior to the open approach to treat lung cancer?" remained unanswered. Moreover, the question why singleincision VATS should be preferred to standard VATS seems to add no value at the more important existing question: is VATS superior to open surgery? [12]. Nevertheless, VATS should be the standard for early-stage lung cancer as it results in minimal morbidity with no compromise in long-term survival [11].

\section{Which VATS technique is preferable?}

The very recent published critical overview says truthfully the true color of the real situation [4]: it is evident that there is no VATS technique which is superior to another to treat lung cancer in terms of longer survival or improved chest pain score. Nowadays it is hard to suggest one VATS technique as the preferred method to perform major lung resection for lung cancer. For the same reasons, the surgeon should choose the VATS method that best suits him [13]. To note, in contrast with the proponents that VATS should be performed only in high-volume units, we have demonstrated that VATS seems to be an effective and safe approach to perform major lung resection in a low-volume unit formed by a single surgeon with a previous high-volume experience [14].

\section{Future research}

Many points will need clarification in the future. Even if not scientifically proven, seeing that the operation to treat lung cancer performed via all existing VATS techniques or open surgery is the same, it appears unclear why VATS should guarantee longer survival than open surgery. Large number of patients will be necessary to prove longer survival rates and therefore it has been suggested that large randomized controlled trials are necessary to compare VATS (every VATS technique to open surgery), to open surgery [15].

Two thoracic groups have demonstrated that the aid of $3 \mathrm{D}$ reconstructions, of the lung and heart, are very useful to obtain preoperative information before VATS surgery [16,17]. The $3 \mathrm{D}$ reconstruction could also forecast a reliable body reconstruction for futuristic self-determining robotic surgery. Robots could start and finish the operation without a human guiding the scalpel with intelligence capabilities. Although the intelligent and autonomous robots offer new opportunities, doubts, law and ethical issues need to be evaluated and resolved [7].

A well-documented experience with longterm survival is mandatory [18] because patients are asking for evidence of survival, and not just on one opinion based on not well-documented experience. On this subject I agree with those authors who wrote that the E in EBM (evidence-based medicine) stands for Evidence, not

Box 1. Existing video-assisted thoracic surgery techniques for non-small-cell lung cancer.

- Single incision (uniportal)
- Bi-portal
- 3-4 ports (multi-portal)
- Mini-thoracotomy $(6 \mathrm{~cm})$


for eminence, experience, expertise, eloquence or any other words that have been used to give authority to one or a group of surgeons [19]. As more innovation and research will be necessary, data from national databases [20] could be used within an international cooperation project to start and finish large prospective studies.

The search for minimal invasiveness has a prominent key role in thoracic surgery, but research is vital to reveal the 'true' advantages of surgical innovations [21].
Financial \& competing interests disclosure The main sponsor of the symposium was Johnson \& Johnson with the contribution of the Department of General Surgery and Medical Specialties of the University of Catania. The author has no other relevant affiliations or financial involvement with any organization or entity with a financial interest in or financial conflict with the subject matter or materials discussed in the manuscript apart from those disclosed.

No writing assistance was utilized in the production of this manuscript.

\section{References}

1 Migliore M, Deodato G. Thoracoscopic surgery, video-thoracoscopic surgery, or VATS: a confusion in definition. Ann. Thorac. Surg. 69, 1990-1991 (2000).

2 Treasure T. Videothoracoscopic resection for lung cancer: moving towards a "standard of care". J. Thorac. Dis. 8(8), E772-E774 (2016).

3 Migliore M, Halezeroglu S, Molins L et al. Uniportal video-assisted thoracic surgery or single-incision video-assisted thoracic surgery for lung resection: clarifying definitions. Future Oncol. 12(Suppl. 23), 5-7 (2016).

4 Perna V. Multiportal vs. uniportal videoassisted thoracoscopic lobectomy: a critical overview. Video-Assisted Thoracic Surgery doi:10.21037/vats.2016.08.07 (2016) (Epub ahead of print).

5 Treasure T, De Leyn P. Rethinking N2 disease in the era of uniportal video-assisted thoracic surgery. Future Oncol. 12(Suppl. 23), 23-26 (2016).

6 Lim E, McElnay PJ, Rocco G et al. Invasive mediastinal staging is irrelevant for PET/CT positive $\mathrm{N} 2$ lung cancer if the primary tumour and ipsilateral lymph nodes are resectable. Lancet Respir. Med. 3(9), e32-e33 (2015).

7 Migliore M. Robotic assisted lung resection need further evidence. J. Thorac. Dis. doi:10.21037/jtd.2016.10.89 (2016) (Epub ahead of print).
8 Zhong JS. Professor Takashi Suda: we should not compromise the safety and precision of thoracic surgery. Ann. Transl. Med. 3(10), 144 (2015).

9 Mineo TC, Tamburrini A, Perroni G, Ambrogi V. 1000 cases of tubeless videoassisted thoracic surgery at the Rome Tor Vergata University. Future Oncol. 12(Suppl. 23), 13-18 (2016).

10 Paul S, Isaacs AJ, Treasure T,Altorki NK, Sedrakyan A. Long term survival with thoracoscopic versus open lobectomy: propensity matched comparative analysis using SEER-Medicare database. BMJ 349, g5575 (2014).

11 Andrews WG, Paul S. Techniques of video-assisted thoracoscopic surgery lobectomy and a critical review of published data. Future Oncol. 12(Suppl. 23), 31-33 (2016).

12 Cheng AM, Wood DE. VATS versus open surgery for lung cancer resection: moving beyond the incision. J. Natl Compr. Canc. Netw. 13(2), 166-170 (2015).

13 Migliore M. VATS surgery for anatomical lung resection: a different approach for every surgeon. Video-Assisted Thoracic Surgery doi:10.21037/vats.2016.10.01 (2016) (Epub ahead of print).

14 Migliore M, Calvo D, Criscione A et al. Safety of video-assisted thoracic surgery lobectomy for non-small-cell lung cancer in a low-volume unit. Future Oncol. 12(Suppl.23), 47-50 (2016).

15 Treasure T. Randomized controlled trials are needed to test videothoracoscopy versus thoracotomy for lung cancer lobectomy. Future Oncol. 12(Suppl. 23), 19-22 (2016).

16 Galluzzo A, Fatica F, Caternicchia F et al. 3D CT scan for perioperative identification of anatomical variations of lungs. Future Oncol. 12(Suppl. 23), 27-30 (2016).

17 Migliore M, Calvo D, Criscione A et al. Lung cancer invading a single left pulmonary vein requiring estended pneumonectomy. Future Oncol. 12(Suppl. 23), 55-57 (2016).

18 Di Rienzo G, Surrente C, Lopez C et al. Tips and tricks in video-assisted thoracoscopic surgery lobectomy. Future Oncol. 12(Suppl. 23), 35-38 (2016).

19 Treasure T, Macbeth F, Russel C. If no difference in effectiveness is found between two treatments it may be because the treatments are similarly ineffective. Ann. Transl. Med. 3(14), 201 (2015).

20 Crisci R, Droghetti A, Migliore M, Bertani A, Gonfiotti A, Solli P; on behalf of the Italian VATS group. Video-assisted thoracic lobectomy for lung cancer in Italy: the 'VATS Group' Project. Future Oncol. 12(Suppl. 23), 9-11 (2016).

21 Migliore M. How surgical care is changing in the technological era. Future Sci. OA 2(2), FSO104 (2016). 